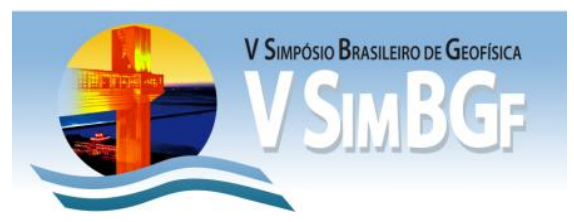

\title{
Modelagem e interpretação de dados magnéticos e gravimétricos de uma seção transversal ao Lineamento Transbrasiliano - Região Sul da Bacia do Parnaíba
}

\author{
Thuany Patrícia C. de Lima* ${ }^{\star *}$ José Antônio de M. Moreira²; Franscisco Hilário R. Bezerra²; Rafael D.Santos; \\ 1-Bolsista da SBGf - Soc. Bras. Geofísica, Departamento de Geofísica - UFRN \\ 2-Programa de Pós-Praduação em Geodinâmica e Geofísica - UFRN \\ 3-Bolsista de doutorado da CAPES pelo Programa de Pós-Graduação em Geodinâmica e Geofísica - UFRN.
}

\section{Copyright 2012, SBGf - Sociedade Brasileira de Geofísica}

Este texto foi preparado para a apresentação no V Simpósio Brasileiro de Geofísica, Salvador, 27 a 29 de novembro de 2012. Seu conteúdo foi revisado pelo Comitê Técnico do V SimBGf, mas não necessariamente representa a opinião da SBGf ou de seus associados. E proibida a reprodução total ou parcial deste material para propósitos comerciais sem prévia autorização da SBGf.

\begin{abstract}
This work proposes a $2.5 \mathrm{D}$ modeling for a section crossing a segment of the Transbrasiliano Lineament in the southern part of Parnaiba Basin, NE Brazil- based on joint inversion of magnetic and gravimetric data. This study points out that the thickness of the sedimentary cover is related to tectonic features such as graben and horsts. Euler deconvolution technique was used in this work aiming to reduce ambiguities of the model as well as to contribute for a better quantification of anomalous source depths. A previous qualitative analysis was necessary to obtain an understanding of different types of sources that might be responsible for magnetic and gravimetric signatures in the area. This analysis was important to a better interpretation of the anomalous sources as long as not all given solutions offered by the Euler technique are those that are aimed to be analyzed.
\end{abstract}

\section{Introdução}

A área de estudo compreende um segmento do Lineamento Transbrasiliano na região Sudeste da Bacia do Parnaíba, NE Brasil. A bacia é considerada do tipo intracratônica e está inserida na Plataforma SulAmericana na Província Parnaíba, possuindo 660.000 $\mathrm{km}^{2}$. No flanco leste, o arcabouço estrutural da Bacia do Parnaíba é fortemente influenciado por uma zona de falhas denominada de Lineamento Transbrasiliano (Schobbenhaus, 1975), que está orientado na direção NE-SW. Esse Lineamento é a feição estrutural mais proeminente na bacia e exerceu influência na formação dos riftes mais antigos da mesma. O Lineamento Transbrasiliano é uma descontinuidade de magnitude continental situada entre o Cráton Amazônico e a porção leste da Plataforma Sul-Americana (Feng et al., 2004). Vários dos falhamentos que cortam as rochas paleozóicas seguem esta orientação. Trabalhos de campo, imagens de satélite, fotografias aéreas e mapa magnético de campo total permitiram identificar estruturas lineares NE-SW na porção leste-sul da Bacia do Parnaíba, que são atribuídas por Góes et al. (1990) ao Lineamento Transbrasiliano. Desta forma, o presente trabalho propõe um modelo magnético-gravimétrico $2.5 \mathrm{D}$ com o objetivo de contribuir para o entendimento da estruturação tectônica em subsuperfície de uma perfil transversal ao Lineamento Transbrasiliano (Fig. 1). A modelagem da seção de direção NW-SE foi subsidiada pelo uso da técnica da deconvolução de Euler. A utilização deste método objetivou reduzir possíveis ambiguidades existentes no modelo. A deconvolução de Euler é uma técnica que fornece uma rápida estimativa da localização e profundidade aparente de fontes potenciais a partir de dados de gravimétrica e magnetometria sem pressupor a direção do vetor de magnetização. A orientação da seção foi escolhida de modo a contemplar os baixos e altos estruturais adjacentes ao lineamento. Existe uma carência de informações de subsuperfície na região de estudo que possa corroborar uma interpretação mais exata do modelo apresentado. No entanto, este trabalho sugere uma primeira estimativa da caracterização das estruturas tectônicas em subsuperfície, criando-se hipóteses a respeito da localização de falhas geológicas presentes da seção, a geometria do topo do embasamento, e ainda a presença de possíveis intrusões não aflorantes. O perfil secciona rochas das Formações Piauí e Pedra de Fogo do Grupo Balsas, e a Formação Potí do Grupo Canindé (Bizzi et al.,2003). Próximo à região ocorre uma sequencia jurássica correspondente às rochas vulcânicas da formação Mosquito, justificando a presença de diques de diabásio no modelo. De acordo com Góes et al. (1992) o diabásio está preferencialmente intrudido no Grupo Canindé (Devoniano-Carbonífero) e não ocupa posição estratigráfica definida.

\section{Dados Gravimétrico e Magnético}

Para realização deste trabalho foram utilizados dados de gravimetria e magnetometria aerocoletados numa área de $750.000 \mathrm{~km}^{2}$, cobrindo partes dos estados do Tocantins, Pará, Maranhão, Piauí, Ceará, Pernambuco e Bahia. Este levantamento foi realizado entre 2005 e 2006 pelo convênio entre a ANP e a Escola Politécnica da Universidade de São Paulo, cobrindo toda a região da Bacia do Parnaíba. As linhas de vôo foram coletadas na direção E-W, espaçadas em $6 \mathrm{~km}$, e as linhas de controle obtidas na direção N-S foram espaçadas em $24 \mathrm{~km}$. Na região de estudo a altura média do vôo foi de $800 \mathrm{~m}$ acima do relevo máximo da área. $O$ processamento dos dados de magnetometria envolveu a remoção da variação diurna, compensação magnética, remoção do IGRF e um micronivelamento, que elimina feições 
espúrias causadas pelas linhas de controle. Nos dados de gravimetria, além das correções usuais de préprocessamento, foram executadas a correção de Eötvös e a correção das acelerações dinâmicas.

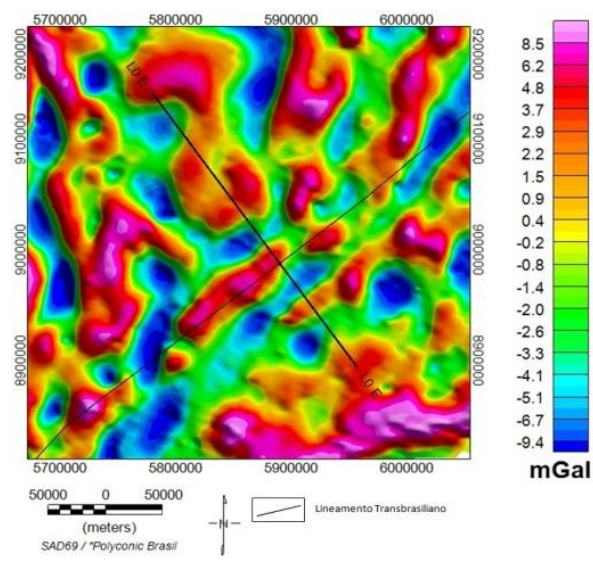

Figura 1: Mapa gravimétrico residual pseudo-iluminado da área de estudo com a localização do perfil modelado(LO E). A linha mais fina com direção SW-NE representa o Lineamento Transbrasiliano. Declinação da fonte de iluminação $45^{\circ} \mathrm{Az}$ e inclinação $45^{\circ}$.

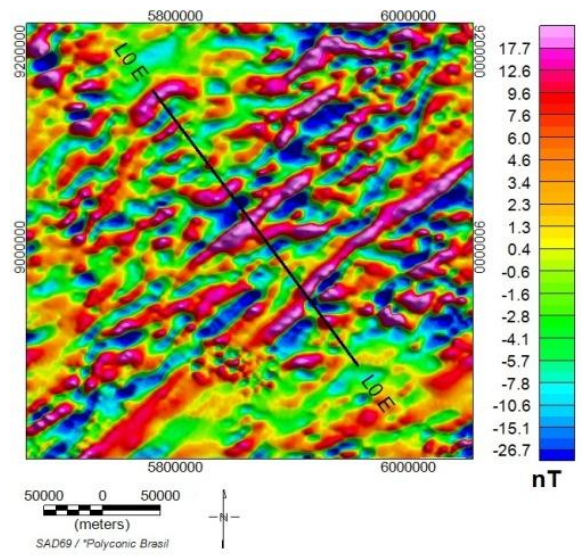

Figura 2: Mapa magnético residual reduzido ao equador pseudo-iluminado. A figura acima contém a localização do perfil modelado (NW-SE). Declinação da fonte de iluminação $45^{\circ} \mathrm{Az}$ e inclinação $45^{\circ}$.

\section{Separação Bouguer regional-residual}

Os dados gravimétricos e magnéticos processados foram interpolados pelo método de mínima curvatura do programa RANGRID do Oasis Montaj (v7.0.1 Geosoft) em uma malha de $1,5 \mathrm{~km} \times 1,5 \mathrm{~km}$, e separados em componentes regional e residual pelo filtro Gaussiano. $\mathrm{O}$ ponto de corte escolhido foi o desvio padrão da função gaussiana, que ofereceu um comprimento de onda de $140 \mathrm{~km}$ (número de onda de 0,007 ciclos $/ \mathrm{km}$ ) para o Mapa Bouguer, e $50 \mathrm{~km}$ (número de onda de $\sim 0,019$ ciclos $/ \mathrm{km}$ ) para o Mapa magnético. As anomalias gravimétricas residuais puderam ser individualizadas de forma satisfatória (Fig.1 e Fig.2). As componentes regional e residual estão relacionadas às fontes de origem profunda e rasa respectivamente.

\section{Deconvolução de Euler}

O método da deconvolução de Euler (Thompson, 1982) é uma ferramenta adicional na interpretação de dados magnéticos e gravimétricos. Trata-se de um método de inversão semi-automática que estima a localização e profundidade aparente de fontes potenciais, indicando mais proveitosamente 0 delineamento de trends e as profundidades de fontes (Reid et al., 1990). A técnica é baseada na equação diferencial homogênea de Euler (1). Abaixo segue a definição da equação de Euler 3D (Reid et al., 1990):

$$
(x-x o) \frac{\partial F}{\partial x}+(y-y o) \frac{\partial F}{\partial y}+(z-z o) \frac{\partial F}{\partial z}=-N F
$$

onde a função homogênea $\boldsymbol{F}$ é o campo potencial observado na posição $(\boldsymbol{x}, \boldsymbol{y}, \boldsymbol{z})$ causada por uma fonte em $(\boldsymbol{x o}, \boldsymbol{y o}, \boldsymbol{z o})$. O $\boldsymbol{N}$, denominado de índice estrutural, é a medida da taxa de mudança do campo potencial com a distância para uma fonte de determinada geometria. De acordo com a literatura, a qualidade das soluções geradas depende essencialmente do índice estrutural $\boldsymbol{N}$ utilizado, ou seja, valores arbitrários de $\boldsymbol{N}$ podem gerar valores enganosos de profundidade. Para a deconvolução foram utilizados os perfis da componente gravimétrica residual e o sinal analítico do campo magnético residual, considerando a altura de voo de 800 m. Para obter as soluções de Euler foi utilizado o software Euler 1.15 (Cooper, 2000,2004) considerando $11 \mathrm{~km}$ o tamanho da janela espacial, na qual todos os seus pontos são usados para o cálculo da resolução da equação de Euler para uma dada posição da fonte. $O$ tamanho da janela escolhido permitiu a distribuição das soluções a uma profundidade de até $6 \mathrm{~km}$ para o perfil magnético e de $8,5 \mathrm{~km}$ para o perfil gravimétrico.

Foram atribuídos os valores do campo total (24810 nT), declinação $\left(-20^{\circ}\right)$ e inclinação magnética $\left(-13^{\circ}\right)$ representativa da área do perfil na época do levantamento. Vários índices estruturais foram testados, mas os que oferecerem melhor cluster de soluções foram: 0,5 e 1 para o perfil magnético e 0 para o perfil gravimétrico. Os outros índices ficaram muito dispersos ou carregados para interpretação, pelo menos para o tamanho de janela $(11 \mathrm{~km})$ utilizada.

Infelizmente, mesmo com o índice estrutural adequado para o tipo de fonte que se procura, parte das soluções oferecidas pela deconvolução é ruído. Desta forma, a análise das soluções de Euler exige muito do interprete, e para não levar a interpretações enganosas, deve ser aplicada com bastante critério.

\section{Modelo magnético-gravimétrico 2D}

A seção modelada apresenta direção NW-SE e possui $303 \mathrm{~km}$ de extensão (Fig. 3). O modelo magnéticogravimétrico $2 \mathrm{D}$ foi produzido fazendo uso da ferramenta GM-SYS (NGA, 2004 ) do software Oasis Montaj (v.7.0.1, 
Geosoft). Os métodos usados para o cálculo das respostas do modelo gravimétrico e magnético são baseados nos métodos de Talwani et al. (1959) ,Talwani \& Heirtzler (1964) e também fazem uso dos algoritmos descritos por Won \& Bevis (1987). A modelagem é efetuada criando-se polígonos fechados de característica homogênea e isotrópica, proporcionando desta forma um ajuste de modelos pelo cálculo das respostas gravimétrica e magnética coerente com o modelo geológico bidimensional imposto. A cada bloco foram atribuídos valores constantes de densidade $e$ susceptibilidade magnética. A ausência de dados de poço na região da área de estudo impossibilitou a atribuição acurada das densidades dos blocos modelados. Os Valores de densidade adotados foram: $2,7 \mathrm{~g} / \mathrm{cm}^{3}$ para o embasamento (Dobrin \& Savit, 1988; Sharma, 1997; Telford et al., 1998); e em média $2,75 \mathrm{~g} / \mathrm{cm}^{3}$ para os diques de diabásio (Telford et al., 1990). Para o contraste de densidade entre o embasamento cristalino e as rochas sedimentares da bacia do Parnaíba foi assumido o valor de $0,35 \mathrm{~g} / \mathrm{cm}^{3}$.

$\mathrm{Na}$ região de trabalho, como a inclinação magnética é muito baixa, utilizou-se para modelagem magnética 0 campo magnético residual reduzido ao equador. Para a redução foram considerados a inclinação magnética da região do perfil $l=-13^{\circ}$, declinação $D=-20^{\circ}$ e campo total 24810nT. O filtro de redução ao equador facilita a interpretação de dados magnéticos por considerar a inclinação do campo magnético igual a zero, e às fontes, o vetor de magnetização zero. Como resultado, concentram-se os picos de mínimos valores sobre fontes de maior susceptibilidade magnética. Considerando então apenas a presença de magnetização induzida, obteve-se o modelo magnético. Para as intrusões básicas as variações de susceptibilidade magnética são da ordem de frações decimais, com valor preponderante atribuído da ordem de $10^{-2}$ c.g.s. Às rochas sedimentares atribuiuse valor zero, e para o embasamento, valores da ordem de $10^{-3}$ c.g.s (Carmichael, 1982).

\section{Discussões}

No presente trabalho, optou-se por utilizar o mapa bouguer residual (Fig. 1) para assegurar a modelagem de fontes em níveis crustais. Ao longo do perfil detectaramse quatro baixos gravimétricos de até $47 \mathrm{~km}$ de comprimento de onda que foram relacionados a baixos estruturais. A anomalia gravimétrica residual mínima presente no perfil é de $-6,08 \mathrm{mGals}$, e ofereceu uma profundidade de $2,13 \mathrm{~km}$ para o topo do embasamento, não ultrapassando a espessura máxima já perfurada de $3,5 \mathrm{~km}$ próximo à região mais central da bacia (Góes et al., 1990). Os outros baixos estruturais interpretados possuem espessuras sedimentares semelhantes entre $1,91 \mathrm{~km}$ e $2,11 \mathrm{~km}$. Às anomalias positivas, por sua vez, foram atribuídos altos estruturais e presença de intrusões básicas não aflorantes. As intrusões modeladas como diques exerceram, em linhas gerais, pouca influencia na delineação da geometria da bacia, que foi previamente modelada apenas com o perfil gravimétrico residual.
A presença dos diques influenciou diretamente na modelagem do perfil magnético. Apesar de no modelo criado através da ferramenta GM-SYS a relação dos picos magnéticos terem sido relacionados majoritariamente à presença de diques, vale salientar que tais anomalias podem estar sendo causadas também pela presença de falhas e pelo contato entre corpos diversos, desde que estes apresentem algum contraste de susceptibilidade. As soluções da Deconvolução de Euler, por sua vez, conseguem identificar esses tipos de fonte, podendo desta forma ajudar a definir o modelo de forma mais precisa, bem como diminuir ambiguidades de interpretação. Durante o processo da modelagem, diferentes índices estruturais foram relacionados ao modelo magnético-gravimetrico. Esses índices (ou grau de homogeneidade) variam com a complexidade das fontes potenciais, e mesmo fazendo uso do valor adequado para o tipo de fonte que se procura, parte das soluções oferecida é ruído.

$\mathrm{Na}$ figura 3 está ilustrado as nuvens de solução de Euler para o índice estrutural 1 aplicado ao dado magnético. Esse índice ajudou na localização e delimitação de espessuras de alguns dos diques modelados. Outros índices estruturais testados ofereceram soluções mais carregadas ou muito dispersas, dificultando o processo de interpretação. Na figura 4, apesar da dificuldade na interpretação, puderam-se perceber algumas tendências de soluções que estão em comum com a geometria do embasamento. Neste caso o índice de 0,5 sugeriu a existência de falhas limitando os altos e baixos estruturais. Nas regiões próximas de $120 \mathrm{~m}$ e $230 \mathrm{~m}$ do perfil existem baixos magnéticos expressivos que podem estar relacionados às falhas geológicas. $O$ índice estrutural 0 aplicado ao dado gravimétrico também indica alguns alinhamentos de falhas (Reid, 2003), entretanto de forma mais insatisfatória.

\section{Conclusões}

Apesar dos resultados ambíguos sugeridos pelos procedimentos matemáticos, o processo de inversão permitiu localizar e inferir características geométricas de feições geológicas em subsuperfície numa seção transversal ao LT na Bacia do Parnaíba. A análise conjunta dos métodos potenciais de gravimetria e magnetometria, subsidiada pelo método da deconvolução de Euler, permitiu a identificação de graben e horsts a partir do contraste de densidade e susceptibilidade magnética inferidos ao modelo. A utilidade da deconvolução de Euler é baseada na identificação de fontes anômalas pela distribuição espacial das soluções. Este método foi utilizado com o objetivo de reduzir possíveis ambiguidades da modelagem.

O modelo apresentado sugere informações sobre feições estruturais em subsuperfície, a saber: falhas delimitadoras dos baixos estruturais da bacia que podem fazer parte do conjunto de falhas NE-SW relacionadas à reativação do embasamento pré-cambriano (Justo, 2006); intrusões de diabásio não aflorantes provindas do magmatismo básico ocorrido no Eojurássico (Góes \& 
Feijó, 1994); e os limites das estruturas de graben e horsts associadas ao lineamento. A profundidade máxima identificada para o topo do embasamento foi de 2,14 km. O perfil magnético foi ajustado majoritariamente pela variação das espessuras dos diques. Esses diques exerceram, em linhas gerais, pouca influencia na delineação da geometria da bacia, deixando o topo do embasamento ligeiramente mais profundo. A interpretação integrada com as soluções da deconvolução de Euler possibilitou a análise do comportamento dos limites dos altos e baixos estruturais, oferecendo a geometria do topo do embasamento na seção estudada. Como se trata de uma bacia pouco conhecida, a ausência de dados de poço na região da área de estudo impossibilitou uma analise mais acurada dos modelos, comprometendo a real profundidade do embasamento. No entanto, o trabalho realizado oferece uma primeira aproximação da caracterização das estruturas tectônicas em subsuperfície baseada em algumas hipóteses. Os resultados oferecidos pela deconvoluçao quando analisados em conjunto com o modelo magnético-gravimétrico oferecem respostas condizentes com a tectônica da região. Entretanto, estudos posteriores utilizando dados de subsuperfície fazem-se necessário para uma análise mais quantitativa do modelo.

\section{Agradecimentos}

Ao projeto Lineamento Transbrasiliano: Origem, Evolução e influência na sedimentação de Bacias Fanerozóicas (Rede Temática de Estudos Geotectônicos), coordenado pelos Dr. Elton Dantas e Dr. Reinhardt A. Fuck (UnB) e financiado pela Petrobrás. A Agência Nacional de Petróleo (ANP) por ceder os dados geofísicos. T.P.C. de Lima é bolsista de iniciação científica da SBGfSociedade Brasileira de Geofísica, F.H.R. Bezerra é bolsista de produtividade do CNPq, R.D. Santos é bolsista de doutorado da CAPES pelo Programa de PósGraduação em Geodinâmica e Geofísica - UFRN.

\section{Referências}

BIZZI LA, SCHOBBENHAUS C, VIDOTTI RM \& GONÇALVES JH. 2003. Tectônica e Recursos Minerais do Brasil.CPRM, Brasília, Geologia,

CARMICHAEL RS. 1982. Physical properties of rocks andminerals. CRC press Inc, 741.

DOBRIN MB \& SAVIT CH. 1988. Introduction to Geophysical Prospecting.McGraw-Hill Book Co., New York, 867 p.

COOPER GRJ.2000-2004. Euler 1.15 - Euler deconvolution for profile data.School of Geosciences. University of the Witwatersrand, Johannesburg, South Africa [Online]. [Acessado 27 de Julho de 20012]. www.witz.ac.za

FENG M, ASSUMPÇÃO M \& VAN DER LEE S. 2004. Group-Velocity Tomography and Lithospheric S-Velocity of South American Continent. Physics of the Earth and Planetary Interiors. 147: 315-331.
GÓES AMO \&FEIJÓ FJ. 1994. Bacia do Parnaíba. Boletim de Geociências da Petrobras, 8(1): 57-67.

GÓES AMO, TRAVASSOS WA \& NUNES KC.1992. Projeto Parnaíba-Reavaliação da bacia e perspectivas exploratórias. Relatótio interno. Belém, PETROBRÁS.

GÓES AMO, SOUZA JMP \&TEIXEIRA LB.1990. Estagio exploratório e perspectivas petrolíferas da Bacia do Parnaíba. Boletim de Geociências da Petrobrás, 4:55-64.

JUSTO AP.2006.Sistemas orbitais e aéreos aplicados à análise multi-escala de lineamentos na borda sudeste da Bacia do Parnaíba. M.Sc. Dissertação, Programa de Pós graduação em geodinâmica e geofísica, Universidade Federal do Rio Grande do Norte.

NGA. 2004. GM-SYS - Gravity/Magnetic Modeling Software: User's Guide version 4.9. Corvallis, Northwest Geophysical Associate, $101 \mathrm{pp}$.

REID AB. 2003. Short note: Euler magnetic structural index of a thin bed fault. Geophysics.Published electronically, May 2003.

REID AB, JM ALLSOP, H Granser, AJ Millett \& IW Somerton.1990. Magnetic interpretation in three dimensions using Euler deconvolution. Geophysics, 55: 80-91.

REID ABD, FITZ G \& Mclnerney P. 2003. Euler deconvolution of gravity data. SEG Annual Meeting, Dallas, accepted for presentation.

SCHOBBENHAUS C, CAMPOS DA, DERZE GR, ASMUS HE. 1975. Texto Explicativo. Folha Goiás SD.22. In: Schobbenhaus C. (coord.) Carta Geológica do Brasil Milionésimo. Brasília, DNPM.

SHARMA PV. 1997. Environmental and engineering geophysics. Cambridge University Press, 475p.

TALWANI M, WORZEL JL \& LANDISMAN M. 1959. Rapid gravity computations for two-dimensional bodies with the application to the Mendocino submarine fracture zone. Journal of Geophysics Research, 64: 49-59.

TALWANI M \& HEIRTZLER JR. 1964. Computation of gravity anomalies caused by two dimensional structures of arbitrary shapes. Geological Sciences, 1(9): 464-480.

TELFORD WM, GELDART LP, SHERIFF RE \& KEYS DA. 1998. Applied Geophysics. 5 ed., Cambridge University Press, $860 \mathrm{p}$.

THOMPSON DT.1982, EULDPH: A new technique for making computer-assisted depth estimates from magnetic data, Geophysics, (47): 31-37. GÓES AMO, Souza JMP, TEIXEIRA L B. 1990. Estagio exploratório e perspectivas petrolíferas da Bacia do Parnaíba. Boletim de Geociências da Petrobrás, 4:55-64.

WON IJ. \& BEAVIS, M. 1987. Computing the gravitationaland magnetics anomalies due to a polygon: algorithms and fortran subroutines. Geophysics, 52: 232238. 


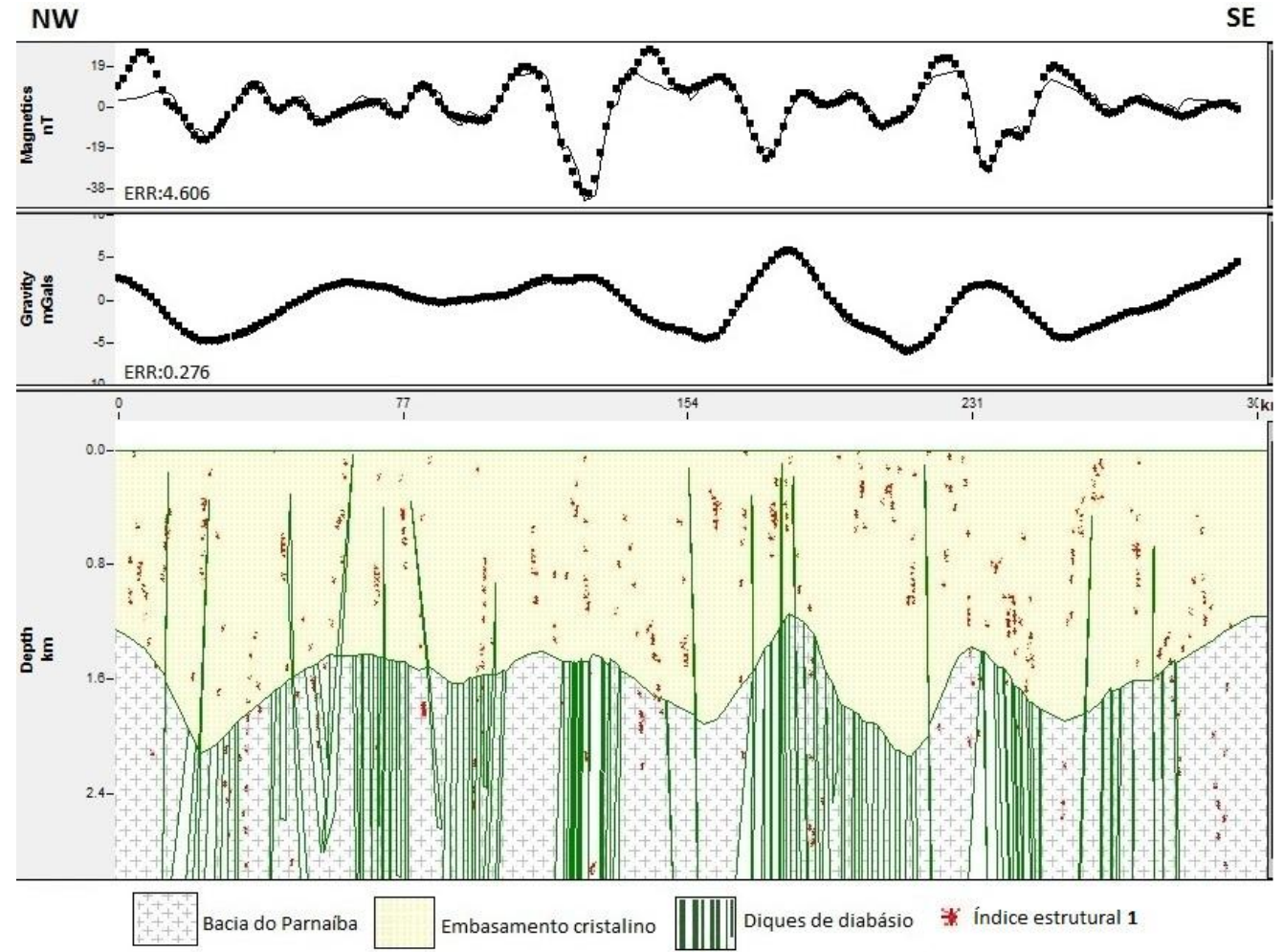

Figura 3: Modelo com nuvem de soluções de Euler do dado magnético com índice estrutural 1. Exagero vertical de 37 vezes.

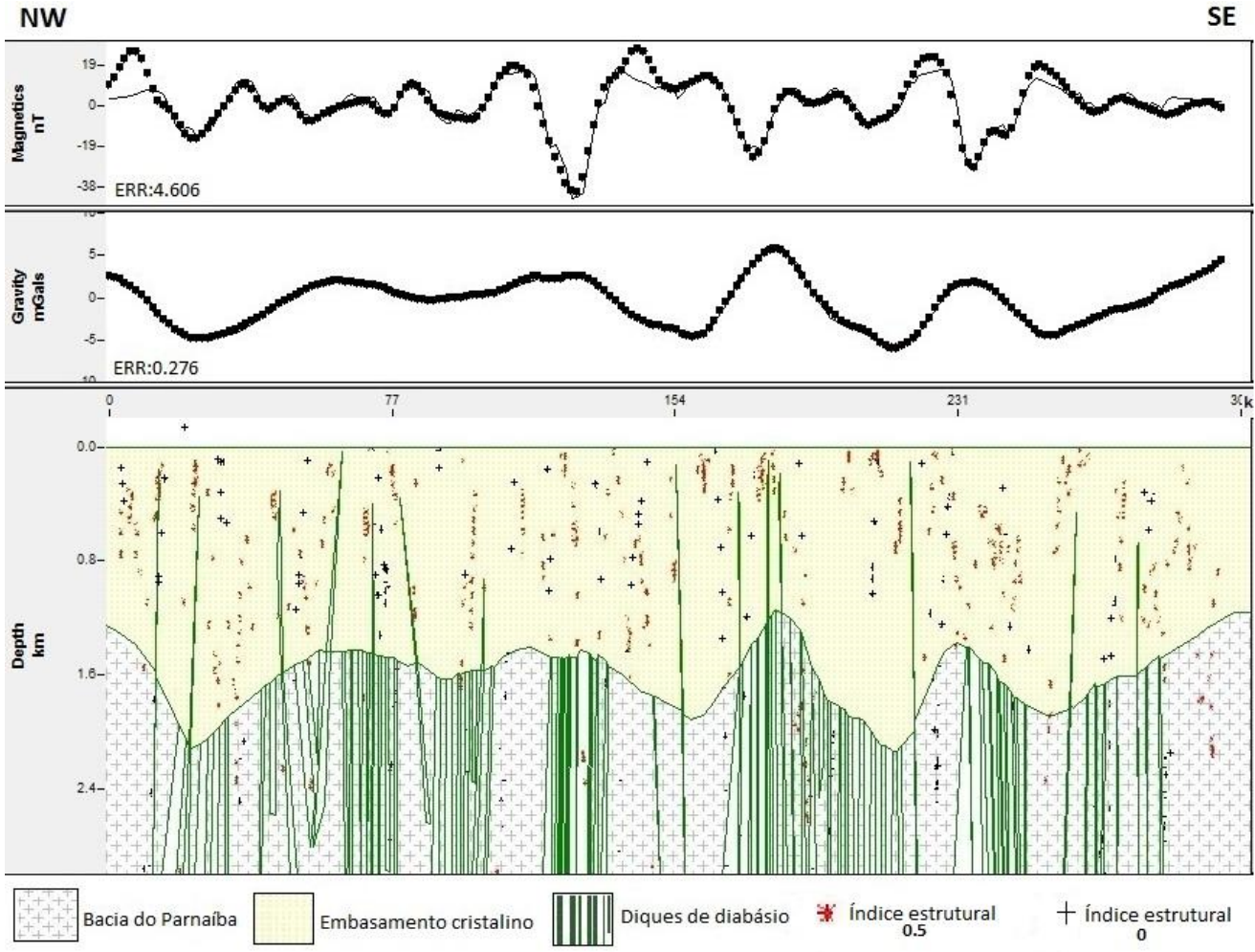

Figura 4: Modelo com nuvem de soluções de Euler do perfil magnético (símbolos vermelhos) e gravimétrico (símbolos pretos) para o índice estrutural 0.5 e 0 respectivamente. Exagero vertical de 37 vezes. 